\title{
PERFIL CLÍNICO-EPIDEMIOLÓGICO DE CHAGÁSICOS ATENDIDOS EM AMBULATÓRIO DE REFERÊNCIA E PROPOSIÇÃO DE MODELO DE ATENÇÃO AO CHAGÁSICO NA PERSPECTIVA DO SUS
}

\author{
Eliane Dias Gontijo, Manoel Otávio C. Rocha e Ulrika Torquato de Oliveira
}

\begin{abstract}
Estsudo caso-controle, para análise de características clínicas e epidemiológicas foi conduzido em 842 indivíduos atendidos em Ambulatório de Referência de Doença de Chagas, em Belo Horizonte, no período de 1985/92. Verificou-se que os chagásicos apresentaram média de idade mais elevada (37,78 anos); menor escolaridade, independente da cor, e desempenhavam atividades de maior esforço $(p<0,05)$. A via vetorial mostrou ser o principal mecanismo de transmissão. As queixas específicas predominaram nos chagásicos $(p<0,05)$. A ausculta cardíaca (OR:2,29 IC95\%: 1,29-4,09) e presença de extra-sístoles (OR:7,16 IC95\%:1,5945,07) foram as alterações mais importantes ao exane clínico. A IFI apressenton maior sensibilidade (94\%), especificidade (96\%); VPP(99\%) e VPN(83\%). A reação de Elisa realizada em 43 indivíduos não mostron falsos resultados. Houve predomínio da FCI(56\%) on formas clínicas iniciais. A importância de realizar propedêutica mais complexa em situações especificas foi reforçada pelo encontro de ECGs normais e alteração em $30 \%$ dos Holter, $41 \%$ dos TE e 33\% dos ECOs, além de $48 \%$ de Rx de tórax normal e ECO alterado. Propõe-se modelo de atenção ao chagásico integrado no SUS valorizando a confirmação sorológica, a preparação de recursos humanos capacitados e comprometidos com o desenvolvimento de ações integrais de saúde e a organização do sistema de referência e contra-referência, garantindo o acesso do chagásico aos vários niveis de atenção.
\end{abstract}

Palavras-chaves: Doença de Chagas. Atenção ao chagásico.

O Programa de Controle da Doença de Chagas desenvolvido pela Fundação Nacional de Saúde, a partir de 1980 , tem sido eficaz no controle de espécies domiciliadas de triatomíneos. Na prática, $80 \%$ da área endêmica da tripanosomíase no Brasil estão sob vigilância, com taxas de infestação domiciliar inferiores a $5 \%^{2}$. Em 1992, o percentual de $T$. infestans capturados no Brasil foi de $1,41 \% \mathrm{e}$ os índices de soro-positividade para $T$. cruzi nos bancos de sangue da Hemorrede, em todo o Brasil, foram de $0,80 \%$. Com a regulamentação do setor e a criação da rede de laboratórios de referência de sorologia para doença de Chagas, considera-se que a maior parte do sangue transfundido, atualmente, no Brasil, esteja sendo submetida à triagem prévia, com a realização concomitante de duas metodologias

\footnotetext{
Ambulatório de Doença de Chagas, Faculdade de Medicina, Universidade Federal de Minas Gerais, Belo Horizonte, MG. Apoio: CENEPI/FNS/MS.

Endereço para correspondência: Profa. Eliane Dias Gontijo. DMPS/FM/UFMG. Av. Alfredo Balena 190, 30130-100 Belo Horizonte, MG. Fax: (031) 222-0300.

Recebido para publicação em 16/12/95.
}

distintas de alta sensibilidade. Mota (1993) acompanhando gestantes atendidas em Hospital Universitário, em Belo Horizonte, no período de 1983 a 1990, detectou a incidencia de $1,8 \%$ de transmissão intra-uterina de doença de Chagas.

Com isso, alcançado o controle da transmissão vetorial e transfusional e considerando a baixa possibilidade da via transplacentária, a questão básica para as autoridades sanitárias concentra-se no atendimento de cinco milhões de chagásicos, destacando-se, a situação desses indivíduos frente ao mercado de trabalho, os benefícios sociais e a necessidade de atenção médica adequada na rede de serviços.

Dados recentes mostraram que em Belo Horizonte, Minas Gerais, em 1989, somente $80 \%$ dos óbitos por doença de Chagas receberam assistência médica, indicando que, provavelmente, a atenção aos doentes menos graves deve ser ainda menor ${ }^{4}$.

A busca de melhor compreensão dos fatores envolvidos neste processo levou-nos a realizar este 
Gontijo ED. Rocha MOC, Oliveira UT. Perfil clinico-epidemiológico de chagásicos atendidos en laboratório de referência proposição de modelo de atençăo ao chagásico na perspectiva do SUS. Revista da Sociedade Brasileira de Medicina Tropical 29:101-108, 1996.

estudo com o objetivo de analisar o perfil de morbidade dos indivíduos atendidos no Ambulatónio de Doença de Chagas e refletir sobre um modelo de atendimento na perspectiva do SUS.

\section{MATERIAL E MÉTODOS}

\section{Área de atuação}

Em 1989, atendendo aos objerivos do subcomponente Atenção ao chagásico do PCDEN/ FNS/MS, a estrutura do Ambulatório d: Doença de Chagas do HC/UFMG, implantado, em 1985, por un dos autores, foi ampliada com o objetivo de assumir as funçôes terciárias do Sistema de Atenção. O financiamento viabilizou a aquisição de equipamentos para realização de ergometria, ecocardiografia e a monitorização eletrocardiográfica ambulatorial contínua Sistema Holter, indispensáveis para uma adequada investigação diagnóstica, neste nível de complexidade. A partir de julho de 1991, esse Serviço passou a integrar o Centro de Treinamento e Referência CTR-DIP, de acordo com o convênio firmado entre a SES/MG e a UFMG visando a integração de esforços no atendimento referencial a pacientes com doenças infecto-parasitárias, na pesquisa desta área de conhecimento e na formação de recursos humanos para o SUS.

\section{População estudada}

Indivíduos atendidos no Ambulatório no período de 1985 e 1992. Casos foram definidos como indivíluos soro-positivos, detectados em pelo menos duas técnicas diferentes. Entre os 909, inicialmente selecionados, 47 (5,2\%) foram excluidos por apresentarem sorologia inconclusiva, tendo sido, então, analisados 842 indivíduos. A maioria dos atendidos ignorava a afecção, considerando-se sadia até o momento da detecção e encaminhamento ao Serviço.

En cada consulta foi preenchido um protocolo padrão, pelo médico assistente, incluindo dados de identificação, dados epidemiológicos, anamnese. exame físico, resultados de exames e conduta. Estas informações, num total de 120 variáveis, foram digitadas e armazenadas no banco de dados Foxbase.
As variáveis estudadas incluíram dados de confirmaçāo sorológica, pelas técnicas de IFI. HAI, ou RFC e a solicitação de ELISA nos casos duvidosos. A reação de fixação de complemento deixou de ser realizada em 1992. Além disso, obteve-se informações de uma série de variáveis relacionadas aos fatores de risco conhecidos da evolução da doença de Chagas, investigação de sintomas e sinais, resultado do ECG convencional em repouso nas doze derivações clássicas, radiografia de tórax em PA e perfil; esofagograma para pesquisa de retenção de contraste. O enema opaco foi realizado nos casos de obstipação superior a cinco dias e o teste ergométrico, Holter e ecocardiograma somente foram solicitados para esclarecimento de possíveis alterações cardíacas.

\section{Análise estatística}

Na fase preliminar da análise, o banco de dados teve sua consistencia verificada. Para cada uma das variáveis, procurou-se por erros de digitação ou algum valor inconsistente; quando esses ocorriam retornava-se ao prontuário, buscando-se a informação correta. Apesar destes cuidados, nem todos os participantes possuem informação para todas as variáveis.

Para a análise do estudo caso-controle o teste de quiquadrado foi utilizado para verificar diferenças na distribuição de frequëencias, com nível de significância de 0.05 . O reste de Wilcoxon foi utilizado na comparação das idades. A medida bruta de associação entre os fatores de risco e a infecção chagásica fol expressa pelo Odds Ratio (OR) com intervalo de confiança de $95 \%$.

A avaliação dos exames sorológicos foi feita pela estimação de sua sensibilidade, especificidade, valores de predição positiva e negativa.

O desempenho dos exames de avaliação do comprometimento cardíaco nos chagásicos foi analisado através de proporções de concordância.

\section{RESULTADOS}

A Tabela 1 mostra a análise univariada de variáveis utilizadas para a caracterização da clientela. Verificou-se que não houve diferença estatística em relação ao encaminhamento por bancos de sangue 
Gontijo ED, Rocha MOC, Oliveira UT. Perfil clínico-epidemiológico de chagásicos atendidos em laboratório de referência e proposição de modelo de atenção ao chagásico na perspectiva do SUS. Revista da Sociedade Brasileira de Medicina Tropical 29:101-108, 1996.

ou postos de saúde entre chagásicos e não chagásicos. Dos 192 indivíduos encaminhados pela rede, a sorologia não foi confirmada em $29 \%$, e dos 650 procedentes dos bancos de sangue, constatou-se $25 \%$ de não chagásicos. Houve predomínio de homens em ambos os grupos, refletindo a clientela preferencial dos serviços de hemoterapia, de cor parda ou negra $(60 \%)$. Os chagásicos tenderam a ser mais velhos, com média de idade de 37,8 anos e terem menos anos de escolaridade ( $84 \%$ com até quatro anos) $(p<0,00)$. Em relação à residência atual a maioria mora na grande Belo Horizonte (69\% dos chagásicos e $75 \%$ dos não chagásicos, $\mathrm{p}>0,05$ ).

Entre os chagásicos observou-se que $70 \%$ dedicam-se a atividades braçais. Houve diferença significativa entre chagásicos e não chagásicos em relação ao grau de esforço dispendido nas atividades laborativas $(p<0,00)$.

Levantamento feito em 164 chagásicos mostrou que a principal reação ao ser informado da sua condição de soro-positividade foi de preocupação

Tabela 1 - Caracterização da clientela atendida em Ambulatório de Referência em Doença de Chagas.

Belo Horizonte, 1985-92.

\begin{tabular}{|c|c|c|c|c|c|}
\hline \multirow{2}{*}{ Características } & \multicolumn{2}{|c|}{ Chagásicos } & \multicolumn{2}{|c|}{ Não chagásicos } & \multirow{2}{*}{ Valor P } \\
\hline & $n^{\circ}$ & $\%$ & $n^{o}$ & $\%$ & \\
\hline \multicolumn{6}{|l|}{ - Encaminhamento } \\
\hline bancos de sangue & 489 & 78 & 161 & 75 & \\
\hline postos de saúde & 137 & 22 & 55 & 25 & \\
\hline Total & 626 & 100 & 216 & 100 & 0,27 \\
\hline \multicolumn{6}{|l|}{$\cdot$ Sexo } \\
\hline masculino & 444 & 71 & 181 & 84 & \\
\hline feminino & 182 & 29 & 35 & 16 & \\
\hline Total & 626 & 100 & 216 & 100 & 0,00 \\
\hline \multicolumn{6}{|l|}{ Cor } \\
\hline branca & 247 & 40 & 87 & 40 & \\
\hline parda & 268 & 43 & 90 & 42 & \\
\hline negra & 104 & 17 & 38 & 18 & \\
\hline Total & 619 & 100 & 215 & 100 & 0,92 \\
\hline - Média de idade (anos) & 37,78 & & 35,88 & & 0,01 \\
\hline \multicolumn{6}{|l|}{ - Escolaridade } \\
\hline primário & 523 & 84 & 123 & 56 & \\
\hline secundário & 94 & 15 & 87 & 40 & \\
\hline superior & 4 & 1 & 9 & 4 & \\
\hline Total & 621 & 100 & 219 & 100 & 0,00 \\
\hline \multicolumn{6}{|l|}{$\begin{array}{l}\text { Classificação do } \\
\text { esforço/trabalho }\end{array}$} \\
\hline leve & 102 & 36 & 71 & 55 & \\
\hline moderado & 125 & 44 & 37 & 28 & \\
\hline pesado & 58 & 22 & 22 & 17 & \\
\hline Total & 285 & 100 & 130 & 100 & 0,00 \\
\hline \multicolumn{6}{|l|}{ - Residência atual } \\
\hline Belo Horizonte & 427 & 69 & 164 & 75 & \\
\hline outros & 194 & 31 & 55 & 25 & \\
\hline Total & 621 & 100 & 219 & 100 & 0,08 \\
\hline
\end{tabular}


Gontijo ED, Rocha MOC, Oliveira UT. Perfil clínico-epidemiológico de chagásicos atendidos em laboratório de referência e proposição de modelo de atenção ao chagásico na perspectiva do SUS. Revista da Sociedade Brasileira de Medicina Tropical 29:101-108, 1996.

(41\%) e tristeza (17\%), medo de morrer (10\%). As principais dúvidas levantadas pelos pacientes incluíram as ligadas aos mecanismos de transmissão e evolução da doença ( $21 \%$ ) e possibilidade de cura $(20 \%)$.

A via vetorial representa o mais importante mecanismo de infecção, com $90 \%$ dos chagásicos relatando terem nascido em zona rural (OR:6,96 IC95\%:4,64-10,47) e $81 \%$ em área endêmica (OR:5,79 IC95\%:4,05-8,30). Constatou-seque 7\% dos chagásicos ainda moram em zona rural.

A história familial positiva com relato de cardiopatia (OR:1,55 IC95\%:1,06-2,25) e morte súbita (OR:2,66 IC95\%:1,79-3,96) em familiares com menos de 40 anos, reforçam o mecanismo vetorial de transmissão além de demonstrar a importância do atendimento se estender ao grupo familiar. Não houve diferença significativa em relação à recepção de sangue ou relato da mãe ser chagásica. Os não chagásicos tenderam a um maior número de doações $(p<0,00)$, demonstrando uma eficácia da triagem sorológica, pelo menos na Hemorrede, principal local de encaminhamento dos indivíduos estudados. Deve-se ressaltar que somente $20 \%$ dos chagásicos nunca doaram sangue.

Os resultados dos testes sorológicos feitos no Hospitas das Clínicas apontam que a imunotluorescência-IFI mostrou os maiores índices de sensibilidade $(94,2 \%)$, especificidade $(96,1 \%)$ e VPP $(98,8 \%)$. A menor sensibilidade foi da reação de fixação de complemento-RFC $(82,43 \%)$ ea menor especificidade da hemaglutinação indiretaHAI $(89,8 \%)$. A garantia que o resultado positivo pertence a um paciente chagásico, dada pelo VPP, mostrou-se mais elevada na IFI $(98,8 \%)$, seguida da $\operatorname{RFC}(97,9 \%)$ e, por último a HAI $(95.3 \%)$. O teste ELISA, feito em 43 participantes, não apresentou falhas na detecção da doença, o que resultou em valores de sensibilidade, especificidade, VPP e VPN de $100 \%$. No entanto, com tão poucos pacientes, não se pode concluir que esse exame é infalível. mas que apresenta altos valores para as medidas de qualidade de testes clínicos.

Algum tipo de queixa esteve presente nos dois grupos $(p=0,37)$, mostrando que, no presente estudo, não houve viés de aferição. A hipertensão arterial foi detectada sem diferença significativa $(\mathrm{p}=0,65)$. Os sintomas de dispnéia (OR : 1,93 IC95\% : 1,35-2,76), palpitações (OR : 1,65 IC95\% : 1,16-2,35), precordialgia (OR : 1,85 IC95\%:1,09-3,17) e disfagia (OR: 1,82 IC95\%: 1,22-2,74) e obstipação (OR : 2,00 IC95\% : $1,06-3,87)$ predominaram entre os chagásicos. A ausculta cardíaca mostrou uma chance 2,29 vezes maior de estar alterada nos chagásicos (IC95\% : 1,29-4,09), enquanto a presença de extrasístoles tinha um risco 7,16 maior de ser detectada nos soro-positivos.

A Tabela 2 mostra os resultados dos exames complementares realizados em $98 \%$ dos participantes $(p=0,47)$. Alterações evidenciadas nos exames solicitados rotineiramente no Ambulatório, como a radiografia de tórax, eletrocardiograma e esofagograma foram mais freqüentes entre os chagásicos. O ECG alterado mostrou uma chance de 3,22 (IC95\%:2,21-4,70) de pertencer a um infectado. As alterações eletrocardiográficas mais freqüentes foram o $\operatorname{BCRD}(15,3 \%)$, $\operatorname{HBAE}(15,1 \%)$. bradicardia sinusal $(8,5 \%)$, as alteraçôes primárias de repolarização $(7.3 \%)$ e o bloqueio átrio-ventricular $(4,6 \%)$. Estas alteraçóes concordam com as encontradas no Inquérito Eletrocardiográfico Nacional para Doença de Chagas 5 .

As alterações encontradas no exame radiológico do esôfago mostraram-se estatisticamente diferentes nos dois grupos $(p<0,01)$, revelando um risco quase duas vezes maior do chagásico apresentar esofagograma alterado. A prevalência de exames alterados em não chagásicos pode estar demonstrando a presença de outros distúrbios motores que ocorrem na população geral.

Quanto aos exames que somente são solicitados mediante al guma queixa específica, não foi veriticada diferença significativa em relação ao enema opaco $(p=0,85)$, ao ecocardiograma $(p=0,51)$ ou ao Holter $(p<0,20)$. Somente o teste ergométrico $(\mathrm{p}<0,03)$ se comportou de modo diferente nos grupos estudados. evidenciando uma chance 5,3 vezes maior de apresentar alteraçôes yuando realizado em indivíduos infectados. Analisando os chagásicos. a comparação entre todas as alterações eletrocardiográficas e o Holter $(p<0,00)$ mostrou 
Gontijo ED. Rocha MOC, Oliveira UT. Perfil clínico-epidemiológico de chagásicos atendidos em laboratório de referência e proposição de modelo de atenção ao chagásico na perspectiva do SUS. Revista da Sociedade Brasileira de Medicina Tropical 29:101-108, 1996.

Tabela 2 - Exames complementares de pacientes atendidos no Ambulatório de Referência de Doença de Chagas. Belo Horizonte, 1985-92.

\begin{tabular}{|c|c|c|c|c|c|c|}
\hline \multirow{2}{*}{ Exames } & \multicolumn{2}{|c|}{ Chagásicos } & \multicolumn{2}{|c|}{ Não chagásicos } & \multirow{2}{*}{ Valor P } & \multirow{2}{*}{$\begin{array}{l}\text { Odds Ratio } \\
\text { (IC 95\%) }\end{array}$} \\
\hline & $n^{0}$ & $\%$ & $\mathrm{n}^{\mathrm{o}}$ & $\%$ & & \\
\hline Realizados (843) & 628 & 98 & 215 & 98 & 0,47 & \\
\hline Rx tórax ALT (491) & 68 & 19 & 14 & 11 & 0,04 & $\begin{array}{c}1,92 \\
(1,00-3,76)\end{array}$ \\
\hline Esofagograma (453) & & & & & & \\
\hline grau 1 & 93 & 28 & 12 & 10 & & 1,87 \\
\hline grau 11 & 15 & 5 & 1 & 0,8 & 0,01 & $(1,11-3,17)$ \\
\hline Enema ALT $(60)$ & 14 & 28 & 3 & 30 & $0,85^{*}$ & \\
\hline ECG ALT (857) & 292 & 46 & 45 & 21 & 0,00 & $\begin{array}{c}3,22 \\
(2,21-4,70)\end{array}$ \\
\hline ECO ALT (96) & 64 & 71 & 5 & 84 & $0,51^{*}$ & \\
\hline Holter ALT (136) & 78 & 57 & 2 & 29 & $0,20 *$ & \\
\hline T. ergométrico (144) & & & & & & \\
\hline insuficiente (27) & 26 & 14 & 1 & 10 & $0,03 *$ & 5,29 \\
\hline alterado (68) & 65 & 49 & 2 & 20 & & $(0,94-38,9)$ \\
\hline
\end{tabular}

* exames realizados mediante sintomas/sinais

uma concordância de $73 \%$, com $13(30 \%)$ indivíduos com ECG normal, mas Holter alterado. Entre o ECG e teste ergométrico $(\mathrm{p}<0,00)$ detectou-se concordância de $72 \%$ e $18(41 \%)$ chagásicos com ECG normal e TE alterado.

Não houve diferença significativa entre o ECG e o ECO $(p=0,43)$. A concordância foi baixa, estimada em $44 \%$, evidenciando-se 13 (33\%) chagásicos com ECG normal e ECO alterado. Por outro lado, o Rx de tórax demonstrou uma diferença significativa $(\mathrm{p}<0,02)$ quando comparado com o ECO, apontando uma concordância de $48 \%$. Importante ressaltar a verificação de 40 indivíduos com radiografia normal e alterações no exame ecocardiográfico (48\%).

Avaliando 160 chagásicos atendidos no biênio $85 / 86$ e reavaliados em $90 / 91$ observou-se que entre 69 pacientes portadores da forma crônica indeterminada, $19 \%$ passaram a apresentar esofagopatia e $25 \%$ evoluíram para cardiopatia. Em relação aos 60 pacientes com forma cardíaca inicial, verificou-se que $13 \%$ apresentaram piora do quadro.

\section{DISCUSSÃO}

O perfil epidemiológico do chagásico é de um indivíduo adulto jovem, de origem rural, infectado por via vetorial, com baixa escolaridade e vivendo em bairros periféricos dos grandes centros urbanos, onde se dedica a ocupações braçais.

A questão de confirmação sorológica da infecção reveste-se da maior importância. Os presentes dados apontam três tipos de problemas relacionados: a ocorrência de $5 \%$ de sorologias inconclusivas, que persistem com reatividade em apenas um teste; 0 encaminhamento pelos bancos de sangue de $25 \%$ de doadores inaptos, rejeitados pelas reações de alta sensibilidade utilizadas para garantir transfusões seguras e, por último, o encaminhamento de $29 \%$ de falso-positivos pelos postos de saúde.

A rede de serviços deve estar apta a enfrentar o dilema de se definir corretamente se estes indivíduos estão ou não infectados. A execução de teste clínicos e laboratoriais adicionais, certamente eleva o custo do atendimento, maséimprescindível para minimizar 
Gontijo ED, Rocha MOC, Oliveira UT. Perfil clínico-epidemiológico de chagásicos atendidos em laboratório de referência e proposição de modelo de atenção aci chagasico na perspectiva do SUS. Revista da Srociedade Brasileira de Medicina Tropical 29:101-108, 1996.

o sof rimento emocional e social imposto ao individuo e sua família. Além disso, é fundamental para o aconselhamento e mesmo a reabilitação de grande parte destes doadores. Por outro lado, o encaminhamento de quase $30 \%$ de falso-positivos pelos postos demonstra o desconhecimento, de muitos profissionais, do manejo adequado do paciente chagásico.

A comparação com grupo de não chaquásicos demonstrou que deve-se valorizar a anamnese e a ausculta cardíaca. As queixas relacionadas à doença, em seus vários acometimentos, foram significativamente diferentes das relatadas pelos não chagásicos e no exame físico, o risco de apresentar uma ausculta cardíaca alterada ou extrasístoles é, respectivamente, 2,3 e 7,2 vezes maior em relação aos não chagásicos.

A solicitação da propedêutica básica incluindo $\mathrm{ECG}, \mathrm{Rx}$ de tórax e esofagograma mostrou-se importante na avaliação do quadro clínico, com diferença significativa quando comparado com os resultados apresentados pelos soro-negativos. Houve predomínio da forma crônica indeterminada (56\%) ou de formas iniciais. Quadros de insuficiência cardíaca ocorreram em menos de $2 \%$ dos participantes.

A deteç̧ão de pacientes com ECGs normais e alterações evidenciáveis em exames propedêuticos mais sofisticados, como o Holter (30\%), teste ergométrico (41\%) e ECO (33\%), assim como a ocorrência de $48 \%$ de pacientes com Rx de tórax normal mas, apresentando algum tipo de alteração ao ecocardiograma, além da evolução de $13 \%$ de cardiopatas para formas mais graves, demonstra a relevância de se desenhar estratégias e estabelecer açōes programáticas para as necessidades identificadas. capazes de atender o paciente ou encaminhá-lo devidamente, mediante mecanismos formalizadores de referência e contra-referência.

Diagnosticado um chagásico, espera-se que o sistema de saúde esteja capacitado a prover o tratamento e acompanhamento do paciente, incluindo a reabilitação, seja a nível do cuidado primário, seja nos níveis secundário, terciário e as ações de caráter preventivo.

Paim $(1993)^{\top}$ aponta uma contradição básica em relação à proposta dos Sistema Locais de Saúde, que estabelece como objeto a resolução dos problemas de saúde da população e a opção pela manutenção de um modelo de organização da assistência centrado no consumo individual de consultas, sob a forma de demanda espontâned, individualista e parcializada de cuidado médico.

A atençăo ao chagásico no Sistema Único de Saúde, exige para sua implantação o desenvolvimento de uma ampla rede hierarquizada de serviços que. distribuída geograficamente, segundo a endemicidade da região, deve prover o atendimento ambulatorial básico às intercorrências clínicas de todas as faixas etárias e, concomitantemente, desenvolver ações de saúde pública. As necessidades da população devem ser diagnosticadas pela análise das características da áréa onde o serviço será implantado englobando dados demográficos, de morbimortalidade, incapacidade, do soropositividade em bancos de sangue e levantamento da capacidade operativa de rede de serviços de saúde. Municípios com maior capacidade instalada, com características de polo regional devem se organizar para o atendimento integral do chagásico, em todos os níveis de complexidade para serem utilizados como referência.

A organização das unidades. a partir dessa lógica, exige uma radical alteração doos mecanismos de gestão e de financiamento da rede básical.

Considerando o quadro clínico do chagásico, o atendimento deve contemplar os indivíduos agendados e, ao mesmo tempo, garantir o atendimento das intercorrências e emergências. Não se pode esquecer também a importância do pronto atendimento dos indivíduos sujeitos a acidentes transfusionais e laboratoriais, reagutizações ou reativações e outras infecções recentes, para os quais existe a possibilidade de cura, através do tratamento específico.

A organização da rede de serviços integrados de saúde deve contemplar a articulação dos níveis de atenção com distinta complexidade de atendimento, hierarquizados entre si, com a finalidade de manter a unidade funcional do sistema. Todos os pacientes devem ter acesso aos vários níveis de atenção, que assegure a realização de métodos propedêuticos especializados e sensíveis, incluindo avaliação ergométrica, ecocardiográfica e por eletrocardiografia dinâmica, tratamento adequado, relatório circunstanciado sobre a condição 
Gontijo ED, Rocha MOC, Oliveira UT. Perfil clínico-epidemiológico de chagásicos atendidos em laboratório de referência e proposição de modelo de atençẫo ao chagásico na perspectiva do SUS. Revista da Sociedade Brasileira de Medicina Tropical 29:101-108, 1996.

clínica observada e encaminhamento à Previdência Social, quando cabível.

A hierarquização exige um fluxo e contra-fluxo de pacientes e de informações gerenciais e técnicocientíficas dentro da rede de serviços. Essas últimas, são básicas para a qualidade de serviços e funcionam como elemento motivador da equipe de saúde, especialmente do pessoal técnico.

Para que seja possível a resolução de, pelo menos, oitenta por cento dos problemas dos chagásicos e atendendo o princípio que os pacientes devem sair o mínimo possível de sua região, o nivel primário deve contar com recursos humanos qualificados e apoio diagnóstico e terapêutico para fornecer sorologia de boa qualidade, fazer a avaliação inicial e de seguimento dos pacientes em relação às repercussões orgânicas e funcionais do sistema cárdio-circulatório e digestivo.

Ao nível secundário devem ser encaminhados, em torno de $15 \%$ dos pacientes, com indicação de avaliação cardiológica especializada. Após a definição do quadro e orientação terapêutica, os indivíduos devem retornar ao nível anterior.

O nível terciário tem as atribuições de servir como referência para o encaminhamento de indivíduos dos outros níveis do Sistema (primário e secundário), que necessitem de cuidados de saúde mais complexos ou com maior incorporação de tecnologia. Estima-se que em torno $5 \%$ dos indivíduos demandam um atendimento mais especializado, para esclarecimento de quadros clínicos complicados ou realização de procedimentos cirúrgicos. Cabe ao nível terciário, ainda, a padronização de condutas e normas de aplicação de métodos propedêuticos para uma efetiva avaliação do grau de morbidade possibilitando um controle médico adequado, orientação trabalhista pertinente, além da proposição de critérios que ampliem o grau de cobertura atual, amparando o chagásico grave.

Ao encaminhar o indivíduo, o serviço deve fornecer uma guia de referência contendo sua história clínica, exames realizados, o diagnóstico firmado e um atestado de capacidade laborativa ou incapacidade.

A ação integral à saúde significa que a partir do atendimento de um paciente, as ações são desdobradas para o grupo, com a organização de práticas preventivas coletivas. Neste enfoque, todas as mulheres grávidas, procedentes de áreas endêmicas, devem ser submetidas à sorologia de rotina, durante o pré-natal, além do acompanhamento sorológico e parasitológico dos seus conceptos. A rotina do Ambulatório de Referência inclui solicitar sorologia de todos os filhos de mães chagásicas atendidas, após os seis meses de idade até os 14 anos, com instituição de tratamento específico dos positivos.

A integração interinstitucional é uma característica básica na operacionalização do modelo de Atenção à Saúde na Doença de Chagas, como forma de recuperar a inadequação e a fragmentação das ações, somar recursos materiais e humanos, evitar duplicidade e paralelismo de ações.

A organização de uma rede de Laboratórios, Centros de Diagnóstico por imagens e de registros gráficos garantiria a eficácia das ações voltadas ao indivíduo, aumentando assim o grau de resolutividade, entendida como a capacitação do serviço de dar respostas eficazes aos problemas de saúde encontrados. O apoio laboratorial deve ser articulado através da rede de Laboratórios de Referência em Sorologia de Doença de Chagas, principalmente, para realizar exames comprobatórios de alta especificidade e sensibilidade.

A preparação de recursos humanos constitui uma etapa indispensável da viabilização do SUS justificada face à especificidade do conhecimento técnico demandado e às características da nova abordagem do processo saúde-doença proposta. Tem como objetivo portanto, a formação de equipe multiprofissional bem preparada tecnicamente e comprometida com o desenvolvimento de ações integradas de saúde, capaz de atender o chagásico em sua globalidade que transcende o enfoque tradicional biologicista. Faz parte essencial desse modelo a preocupação com a consciência sanitária dos usários. A informação em saúde se constitui um instrumento essencial para a gestão dos serviços de saúde, reforçada no contexto da atenção aos chagásicos pela interface entre o setor saúde e a Previdência Social. O profissional de saúde, ao atender um chagásico deve informá-lo da doença, os riscos existentes em seu trabalho e o impacto sobre a evolução da doença, além da conscientização para a não doação. Ressalte-se que no presente trabalho, $80 \%$ dos chagásicos relataram pelo menos 
Gontijo ED, Rocha MOC, Oliveira UT. Perfil clínico-epidemiológico de chagásicos atendidos em laboratório de referência e proposição de modelo de atenção ao chagásico na perspectiva do SUS. Revista da Sociedade Brasileira de Medicina Tropical 29:101-108, 1996.

uma doação. É fundamento utilizar a consulta médica como momento privilegiado de prover o chagásico de informação científica decodificada sobre sua doença, o que lhe pode ser útil para transformação e melhoria das condições de vida e trabalho.

\section{SUMMARY}

A case-control study to analyse clinical and epidemiological data of 842 patientes seen in an outpatient ward reference center for chagas disease in Belo Horizonte, Brazil, from 1985 to 1992. It was verified that these patients were in a average age of 37.78 years; lower literacy regardless of race, and, perforned activities that demanded greater muscle effort $(p<0.05)$. The main mechanism for disease transmission proved to be the vectorial route. Disease-especific complaints prevailed in the above mentioned patients $(p<0.05)$. The most important abnormalities found in clinical examination were with cardiac sounds (OR:2.96 95\% CI:I.29-4.09) and the presence of extrasystole (OR:7.16 95\% CI:1 59-45.07). Imunofluorescence Test was more sensitive (94\%) and specific (96\%). It also had a PPV of $99 \%$ and $e N P V$ of $83 \%$. ELISA test carried out in 43 patientes did not yield false results. Indeterninate chronic phase (56\%) or early stages of the disease prevailed. The findings of normal EKGs and abnormalities in $30 \%$ of the Holter tests, $41 \%$ of the exercise tests, $33 \%$ of the ECO tests and, $48 \%$ of normal chest $x$-ray with abnormal $E C O$. high lights the importance of caring out more detailed exams in specific situations. We hereby propose a care model for the Chagas disease patient, integrated to our State Health Plan stressing serologic tests, and training capable hunan resources committed to the development of integrated healthcare and the optimization of our referral and counter-referral system, thus issuing total care to the Chagas disease patient.

Key-words: Chagas' disease. Chagas' disease care.

\section{REFERÊNCIAS BIBLIOGRÁFICAS}

1. Campos GWS, Merhy EE, Nunes ED. Planejamento sem normas. Hucitec, São Paulo, p.134. 1989.

2. Dias JCP. Estratégias e Perspectivas atuais da consolidaçāo do controle de vetores na etapa de vigilância epidemiológica. Revista da Sociedade Brasileira de Medicina Tropical 26:63-65, 1994.

3. Gontijo ED. Atenção ao chagásico no SUS. Revista da Sociedade Brasileira de Medicina Tropical 26:83-85, 1983.

4. Gontijo ED. Dias, JCP. Mortalidade por doença de Chagas em Minas Gerais: análise preliminar do período de 1978-1989. Revista da Sociedade Brasileira de Medicina Tropical 27:119-120, 1994.

5. Macêdo $V$. Inquérito eletrocardiográfico nacional para doença de Chagas. Revista da Sociedade Brasileira de Medicina Tropical 26:12-13, 1993.

6. Mota C. Significado diagnóstico e conduta na doença de Chagas congênita. Revista da Sociedade Brasileira de Medicina Tropical 26:76-78, 1993.

7. Paim J. A reorganização da prática de saúde em Distritos Sanitários. In: Mendes E, (ed) Distrito Sanitário. HUCTEC. São Paulo, p.187-220, 1993. 